\title{
Hashimoto's disease in a cohort of 29 children and adolescents. Epidemiology, clinical course, and comorbidities in the short and long term
}

Eliot Gómez López, Degree in Medicine, ${ }^{a}$ Ana P. Nso-Roca, M.D., ${ }^{b}$ Mercedes Juste Ruiz, M.D. ${ }^{b}$ and Ernesto Cortés Castell, M.D.

\begin{abstract}
Hashimoto's thyroiditis is the most common cause of goiter and hypothyroidism among children and adolescents. Its clinical manifestations and course vary. The objective of this study was to review the clinical and evolutionary characteristics of Hashimoto's disease in the pediatric population.

Clinical, analytical, ultrasound, epidemiological, and evolutionary data of patients with autoimmune thyroiditis seen at Hospital Universitario San Juan de Alicante between January 2010 and January 2016 were analyzed.

A total of 29 patients were included in the study. A higher prevalence of girls was observed, at a 2:1 ratio. The main reason for consultation was the accidental detection of high thyroid stimulating hormone levels or positive anti-thyroid antibodies in a lab test done for other reason. In the initial analysis, 53.3\% of patients had subclinical hypothyroidism and only $10 \%$ of them had frank hypothyroidism. Two patients developed thyroid cancer.

Key words: Hashimoto's disease, autoimmune thyroiditis, hypothyroidism, child.

http: / / dx.doi.org/10.5546/ aap.2018.eng.56
\end{abstract}

To cite: Gómez López E, Nso-Roca AP, Juste Ruiz M, et al. Hashimoto's disease in a cohort of 29 children and adolescents. Epidemiology, clinical course, and comorbidities in the short and long term. Arch Argent Pediatr 2018;116(1):56-59. a. Department of Neurology, Hospital General Universitario Reina Sofía, Murcia (Spain).

b. Department of Pediatrics, Hospital Universitario San Juan de Alicante, San Juan de Alicante, Alicante (Spain).

c. Department of Pharmacology, Pediatrics, and Organic Chemistry, Universidad Miguel Hernández de Elche, San Juan de Alicante, Alicante (Spain).

E-mail address: Ana P. Nso Roca, M.D.: ananso@yahoo.es

Funding: None.

Conflict of interest: None.

Received: 2-21-2017

Accepted: 7-10-2017

\section{INTRODUCTION}

Hashimoto's thyroiditis is the most common cause of thyroid disease among children and adolescents. ${ }^{1-3}$

The histological characteristics of this disease include the presence of a diffuse lymphocytic infiltrate in the thyroid gland accompanied by a variable degree of fibrosis.

Its clinical manifestations at the time of diagnosis and clinical course vary. Most patients have no symptoms and a normal thyroid function at the time of diagnosis. Among symptomatic patients, the most common clinical manifestations are goiter and growth retardation. ${ }^{4}$

This disease is characterized by the presence of anti-thyroid peroxidase (TPO) antibodies and anti-thyroglobulin (TG) antibodies in blood. A thyroid hormone analysis and ultrasound are useful to make a complete diagnosis of these patients.

The objective of this study was to review the clinical and evolutionary characteristics of Hashimoto's disease in a pediatric population.

\section{POPULATION AND METHODS}

Hospital Universitario San Juan de Alicante sees patients from the Department of Health ${ }^{17}$ of the Valencian Community and caters for a population of 220000 inhabitants, with over 33000 children and adolescents younger than 15 years.

A descriptive, retrospective study was done in a cohort of patients diagnosed with autoimmune thyroiditis before 18 years old and seen at the Department of Pediatrics of Hospital Universitario San Juan de Alicante between January 2010 and January 2016.

The study was approved by the hospital's Ethics Committee.

The medical records of patients with final diagnosis of Hashimoto's thyroiditis or disease, autoimmune thyroiditis or hypothyroidism or chronic lymphocytic thyroiditis were included. The diagnosis of these patients was based on the 
presence of anti-thyroid antibodies; their followup included a complete physical examination and a thyroid function test every 6 months and labs, including complete autoimmunity testing, every year. An ultrasound was done initially and then twice-yearly. Different clinical, analytical, and ultrasound outcome measures were analyzed. The most important outcome measures included year and age at the time of diagnosis, sex, pubertal development as per Tanner, ${ }^{5}$ reason for the first consultation, family history of thyroid disease or other autoimmune conditions, associated diseases (whether or not autoimmune), goiter, anti-thyroid antibodies, and thyroid ultrasound.

Patients were classified as euthyroid if they had thyroid stimulating hormone (TSH) $<5 \mu \mathrm{U} /$ $\mathrm{mL}$ with normal free tetraiodothyronine (T4L) (local lab's reference values: $0.7-1.6 \mathrm{pg} / \mathrm{mL}$ ), subclinical hypothyroid if they had TSH $>5 \mu \mathrm{U} /$ $\mathrm{mL}$ with normal $\mathrm{T} 4 \mathrm{~L}$, and frank hypothyroid if they had high TSH and low T4L. ${ }^{6}$

Data were introduced in a database maintaining confidentiality according to Organic Law 15/1999 for Data Protection and Royal Decree 994/99 (Spain).

Data statistical analysis was done using the IBM-SPSS statistics software, version 22.0.

\section{RESULTS}

During the six years of the study, a total of 33 patients were diagnosed with Hashimoto's disease: 29 met the study inclusion criteria; the other 4 were excluded because they did not attend planned checkups.

The demographic and clinical characteristics are described in Table 1. Participants' age ranged from 5 to 15 years (median age: 11 years). The median follow-up was 31.5 months.

Out of the 29 patients included in the study, 26 had an ultrasound image compatible with diffuse thyroid disease and 3 had a normal ultrasound at the time of diagnosis.

Ultrasound measurements of the thyroid were compatible with an enlarged thyroid in 14 patients and a normal thyroid size also in 14 . No ultrasound data were available regarding the thyroid diameter of the remaining patient.

In relation to the level of antibodies in peripheral blood over the follow-up period, 29 patients ${ }^{4}$ showed an increased level, 20 had the same level, and the level of 5 patients went back to normal. In addition, when comparing the TSH level at the time of diagnosis to that at the end of the follow-up period, the median TSH level reduced from 7.2 to 3.28 .

A study was done to determine if the clinical course of thyroid disease was related to the initial reason for consultation that led to patient diagnosis. An improvement was observed among patients whose initial reason for consultation was an accidental finding, and a worsening among those with goiter or clinical manifestations of thyroid disease at initiation. However, it was not possible to demonstrate the statistical significance of this situation (Table 2).

In our study, there were 2 thyroid cancer cases: an 11-year-old girl with follicular thyroid carcinoma and a 10-year-old boy with papillary

TABLE 1. General characteristics, comorbidities, and family history of patients (N: 29)

\begin{tabular}{lc}
\hline Outcome measure & $\begin{array}{c}\text { Frequency: } \\
\text { n (\%) }\end{array}$ \\
\hline Sex & \\
Girls & $19(65.5 \%)$ \\
Boys & $10(34.5 \%)$ \\
Tanner & \\
T1 & $9(31 \%)$ \\
T2-4 & $16(55 \%)$ \\
T5 & $4(14 \%)$ \\
Reason for consultation & \\
Accidental detection of high TSH or & \\
positive autoantibodies & $20(69 \%)$ \\
Goiter & $6(20.5 \%)$ \\
Clinical manifestations compatible & \\
with thyroid disease & $3(10.5 \%)$ \\
Goiter & \\
Yes & $13(45 \%)$ \\
No & $16(55 \%)$ \\
Initial labs & \\
Subclinical hypothyroidism & $16(55 \%)$ \\
Frank hypothyroidism & $3(10.5 \%)$ \\
Subclinical hyperthyroidism & $1(3.5 \%)$ \\
Euthyroidism & $9(31 \%)$ \\
Anti-thyroid antibodies & \\
Anti-TPO & $18(62 \%)$ \\
Anti-TG & $1(3.5 \%)$ \\
Both & $10(34.5 \%)$ \\
Autoimmune comorbidity & $4(13.3 \%)$ \\
Celiac disease & $2(6.7 \%)$ \\
Alopecia areata & $1(3.3 \%)$ \\
Vitiligo & $1(3.3 \%)$ \\
Non-autoimmune comorbidity & $17(56.7 \%)$ \\
Overweight & $6(20 \%)$ \\
Thyroid cancer & $2(6.7 \%)$ \\
Thyroid disease & $15(50 \%)$ \\
Other autoimmune condition & $12(40 \%)$ \\
\hline TSt thyrids & $3(10 \%)$ \\
\hline
\end{tabular}

TSH: thyroid stimulating hormone; anti-TPO: anti-thyroid peroxidase antibodies; anti-TG: anti-thyroglobulin antibodies. 
thyroid carcinoma. Both underwent a total thyroidectomy and showed an adequate clinical course.

\section{DISCUSSION}

Autoimmune thyroiditis is the main cause of acquired hypothyroidism in the pediatric population. ${ }^{1,2}$ It causes significant morbidity and any information available on the development of this disease in children and adolescents will help us improve its management and follow-up to prevent long-term morbidities.

Autoimmune thyroiditis is more prevalent among the female sex. Our study is no exception: the female:male ratio was 2:1. Although the underlying mechanisms are still unknown, it is suspected that hormonal, environmental, and genetic factors are involved. ${ }^{2}$

The median age of patients in our study was 11 years, which is similar to what has been described in previous studies. ${ }^{7}$

In our study, the main reason for consultation was the accidental finding followed by goiter detection during physical examination. The prevalence of goiter in our study was similar to that observed by Skarpa et al. ${ }^{4}$ and lower to that described in other studies, which ranged from $50 \%$ to $100 \% .{ }^{8}$ This is because of the different inclusion criteria used in the different studies.

Among our study patients, only 3 had frank hypothyroidism. The rate of overt hypothyroidism in the different series ranged between $10 \%$ and $52.4 \%, 2,9,10$ and some studies have even found more than $80 \%$ of euthyroidism. ${ }^{11}$ Such variation may be due to differences in diagnostic protocols, the rate of iodine intake, the female:male ratio, and diagnostic criteria among the different studies.

In relation to the presence of anti-thyroid antibodies, a slightly higher rate of anti-TG antibodies was observed among older children, which was not statistically significant. However, these data are consistent with literature reviews, which have stated that younger children with autoimmune thyroiditis have positive anti-TPO antibodies, but less than $50 \%$ are positive for antiTG antibodies; notwithstanding this, it has been observed that both antibody levels remain high among adolescents. ${ }^{1}$

Most patients had an ultrasound image compatible with diffuse thyroid disease and almost one-half had an enlargement of the thyroid. Fava et al. have described an association between compatible ultrasound alterations and a more aggressive disease. ${ }^{2}$

In relation to associated comorbidities, other autoimmune diseases were observed in 4 patients. In this study we detected an association with family history somewhat higher than Demirbilek et al., who found that $40 \%$ of patients had a family history of thyroid disease. ${ }^{12}$

The evolutionary study detected a trend towards an improved clinical course among patients diagnosed due to an accidental finding and a worse clinical course among those who showed clinical manifestations of thyroid disease. Fava et al. ${ }^{2}$ found a similar association.

Studies conducted in adults and children have detected up to $32 \%$ of patients with thyroid cancer and autoimmune thyroiditis. ${ }^{13,14}$ In our study, 2 patients developed thyroid cancer. This may appear in patients with autoimmune thyroiditis, but it has not been demonstrated that thyroiditis is a premalignant state.

This study has certain limitations, such as the small sample size and little follow-up in some patients.

However, the main strength of this study was the inclusion of all patients diagnosed with autoimmune thyroiditis in our area, which provides consistent results for our work field.

TABLE 2. Relationship between the reason for consultation and subsequent clinical course (N: 29)

\begin{tabular}{llcccc}
\hline & & \multicolumn{3}{c}{$\begin{array}{c}\text { Reason for consultation } \\
\mathbf{n ~ ( \% )}\end{array}$} \\
\cline { 3 - 6 } & & Accidental finding & Goiter & $\begin{array}{c}\text { Clinical manifestations } \\
\text { of thyroid disease }\end{array}$ \\
\hline Clinical course & Worsening & $1(5.26 \%)$ & $1(16.6 \%)$ & $2(50 \%)$ & 0 \\
& Improvement & $5(26.3 \%)$ & 0 & $2(50 \%)$ & 5 \\
& Stability & $13(68.4 \%)$ & $5(83.4)$ & 4 & 20 \\
\cline { 3 - 6 } Total & & 19 & 6 & 29 \\
\hline
\end{tabular}

$\mathrm{p}=0.097\left(\chi^{2}\right.$ test $)$. 


\section{Acknowledgments}

We would like to thank Marta Díaz Nieto, B.S. in Education, specialized in English, for her collaboration reviewing the abstract.

\section{REFERENCES}

1. LaFranchi S. Tiroiditis. En Kliegman R, editor. Nelson: Tratado de Pediatría. 19. a ed. Barcelona: Elsevier; 2012: 1975-7.

2. Fava A, Oliverio R, Giuliano S, et al. Clinical Evolution of Autoimmune Thyroiditis in Children and Adolescents. Thyroid 2009;19(4):361-7.

3. Caturegli P, De Remigis A, Chuang K, et al. Hashimoto's thyroiditis: celebrating the centennial through the lens of the Johns Hopkins hospital surgical pathology records. Thyroid 2013;23(2):142-50.

4. Skarpa V, Kousta E, Tertipi A, et al. Epidemiological characteristics of children with autoimmune thyroid disease. Hormones (Athens) 2011;10(3):207-14.

5. Marshall WA, Tanner JM. Variations in pattern of pubertal changes in girls. Arch Dis Child 1969;44(235):291-303.

6. Mayayo E, Ferrández Longás A, Labarta JI. Interpretación de las pruebas tiroideas. An Esp Pediatr 2002;56(Supl 4):42-52.
7. Alos N, Huot C, Lambert R, et al. Thyroid scintigraphy in children and adolescents with Hashimoto disease. J Pediatr 1995;127(6):951-3.

8. De Vries L, Bulvik S, Phillip M. Chronic autoimmune thyroiditis in children and adolescents: at presentation and during long-term follow-up. Arch Dis Child 2009;94(1):33-7.

9. Doeker B, Reinehr T, AndlerW.Die autoimmunthyreoiditis bei kindern und jugendlichen: linische und biochemische befunde bei 34 patienten. Klin Pediatr 2000;212(3):103-7.

10. Rother K, Zimmerman D, Schwenk F. Effect of thyroid hormone treatment on thyromegaly in children with Hashimoto disease. J Pediatr 1994;124(4):599-601.

11. Rallison ML, Dobyns BM, Keating FR, et al. Occurrence and natural history of chronic lymphocytic thyroiditis in childhood. J Pediatr 1975;86(5):675-82.

12. Demirbilek H, Kandemir N, Gonc EN, et al. Hashimoto's thyroiditis in children and adolescents: a retrospective study on clinical, epidemiological and laboratory properties of the disease. J Pediatr Endocrinol Metab 2007;20(11):1199-205.

13. Sclafani AP, Valdes M, Cho H. Hashimoto's thyroiditis and carcinoma of the thyroid: optimal management. Laryngoscope 1993;103(8):845-9.

14. Hung W, Anderson KD, Chandra RS, et al. Solitary thyroid nodules in 71 children and adolescents. J Pediatr Surg 1992;27(11):1407-9.

\title{
Osteomyelitis in burn children: Ten years of experience
}

\author{
María T. Rosanova, M.D. ${ }^{a}$, Carla Voto, M.D. ${ }^{a}$, Susana Carnovale, M.D. ${ }^{c}$, Nidia Tramonti, M.D. ${ }^{b}$, \\ Jimena Lema, M.D. ${ }^{b}$, José L. Pinheiro, Biochemist ${ }^{c}$, Adela Isasmendi, M.D. ', Verónica Álvarez, B.S. ${ }^{a}$, \\ Rosa M. Villasboas, M.D. ${ }^{b}$, Santiago Laborde, M.D. ${ }^{b}$ and Hugo Basilico, M.D. ${ }^{b}$
}

\begin{abstract}
Osteomyelitis is uncommon among burn patients.

Objective: To describe the clinical, microbiological, and evolutionary characteristics of burn children with osteomyelitis hospitalized in a tertiary care facility.

Methods: Retrospective and descriptive study conducted between January 2007 and January 2017.

Results: Out of 600 burn children, 12 developed osteomyelitis (incidence: $2 \%$ ). Eleven patients had a burn caused by direct fire. Patients' median age was 42.5 months (interquartile range [IQR]: 27-118 months), and their median burned surface area
\end{abstract}

a. Department of Epidemiological Control and Infectious Diseases.

b. Plastic Surgery and Burn Unit.

c. Department of Microbiology.

Hospital de Pediatría "Prof. Dr. Juan P. Garrahan,"

Buenos Aires, Argentina.

E-mail address:

María T. Rosanova, M.D.: margris2@yahoo.com.ar

Funding: None.

Conflict of interest: None.

Received: 4-11-2017

Accepted: 7-19-2017 was 33.5\% (IQR: 18.5-58\%). Osteomyelitis was diagnosed at a median period of 30 days following the burn injury. The most common locations were the upper limbs and the cranial vault. Fever was the most frequent clinical manifestation. The most common microorganisms isolated in bone tissue were fungi in 9 patients. All showed compatible anatomopathological findings. The treatment lasted a median of 44.5 days (IQR: 34.5-65.5 days). Six patients had motor sequelae and 1 died. Conclusion: Fungal osteomyelitis was the most commonly observed etiology. Half of patients had functional sequelae and only 1 patient died.

Key words: osteomyelitis, burn, child.

http: / / dx.doi.org/10.5546/ aap.2018.eng.59

To cite: Rosanova MT, Voto C, Carnovale S, et al. Osteomyelitis in burn children: Ten years of experience. Arch Argent Pediatr 2018;116(1):59-62.

\section{INTRODUCTION}

Osteoarticular infections secondary to burns are infrequent in the field of pediatrics. ${ }^{1}$ The extent of the burn surface area and burn depth, together with exposure of the structures beneath the skin, are predisposing factors. ${ }^{1,2}$ The clinical manifestations are subtle and differ from those of healthy hosts. An early diagnosis and treatment of 